\title{
Mycobacterium chimaera pulmonary infection complicating cystic fibrosis: a case report
}

\author{
Stéphan Cohen-Bacrie ${ }^{1,2}$, Marion David ${ }^{3}$, Nathalie Stremler ${ }^{3}$, Jean-Christophe Dubus ${ }^{3}$, Jean-Marc Rolain ${ }^{1,2}$ and \\ Michel Drancourt ${ }^{1,2^{*}}$
}

\begin{abstract}
Background: Mycobacterium chimaera is a recently described species within the Mycobacterium avium complex. Its pathogenicity in respiratory tract infection remains disputed. It has never been isolated during cystic fibrosis respiratory tract infection.

Case presentation: An 11-year-old boy of Asian ethnicity who was born on Réunion Island presented to our hospital with cystic fibrosis after a decline in his respiratory function over the course of seven years. We found that the decline in his respiratory function was correlated with the persistent presence of a Mycobacterium avium complex organism further identified as M. chimaera.

Conclusion: Using sequencing-based methods of identification, we observed that $M$. chimaera organisms contributed equally to respiratory tract infections in patients with cystic fibrosis when compared with M. avium subsp. hominissuis isolates. We believe that M. chimaera should be regarded as an emerging opportunistic respiratory pathogen in patients with cystic fibrosis, including young children, and that its detection warrants longlasting appropriate anti-mycobacterial treatment to eradicate it.
\end{abstract}

\section{Introduction}

Mycobacterium avium complex (MAC) organisms are opportunistic pathogens, and their isolation from the respiratory tract of cystic fibrosis $(\mathrm{CF})$ patients correlates with declining respiratory function [1]. Six of nine MAC species have been described in the past five years [2-4], but the spectrum of MAC organisms infecting CF patients is not well established; only M. avium and Mycobacterium intracellulare have been reported in these patients $[1,5]$.

Our reference mycobacteriology laboratory routinely processes respiratory tract specimens collected from all CF patients who are followed by the Cystic Fibrosis Regional Reference Center in Marseilles, France. After Ziehl-Neelsen staining, cultures were routinely performed in BACTEC broth using the BACTEC $9000 \mathrm{MB}$ system (BD Biosciences, Sparks, MD, USA), subcultures were grown on $5 \%$ sheep blood agar, and identification

\footnotetext{
* Correspondence: michel.drancourt@univmed.fr

'Unité de Recherche sur les Maladies Infectieuses et Tropicales Emergentes, CNRS-UMR 6236, IRD 198, IFR 48, Faculté de Médecine, Université de la

Méditerranée, 27, Boulevard Jean Moulin, Marseille cedex 5, France Full list of author information is available at the end of the article
}

was done by partial rpoB-based sequencing [2]. Genotyping of the isolates was performed using multi-spacer sequence typing (MST) [6]. The minimum inhibitory concentrations were determined by 10-day incubation of a $1 \mathrm{McF}$ arland mycobacterial suspension at $37^{\circ} \mathrm{C}$ on $5 \%$ sheep blood agar in the presence of the respective Etest strip (AB Biodisk, Solna, Sweden). The susceptibility to rifabutin was determined in BACTEC broth using the BACTEC $9000 \mathrm{MB}$ system by incubating a 1 McFarland bacterial suspension with $1 \mu \mathrm{g} / \mathrm{mL}$ or $2 \mu \mathrm{g} / \mathrm{mL}$ rifabutin for five days in the presence of a growth control.

Over a six-year period, five (4.3\%) of 115 children with $\mathrm{CF}$ had at least one respiratory tract specimen that was positive for MAC. M. avium subsp. hominissuis was found in two patients (with distinct MST48 and MST20 genotypes), Mycobacterium colombiense was found in one patient, and Mycobacterium chimaera was found in two additional patients (distinct MST33 and MST39 genotypes).

\section{Case presentation}

One of the latter five patients with CF described at the end of the Introduction was an Asian boy born on

\section{C)


Réunion Island who exhibited a $\Delta$ F508 homozygous deletion in the CFTR gene, which is diagnostic of CF. He presented to our hospital when he was 11 years old because he had started complaining of hemoptoic cough. A physical examination revealed weight loss and wheezing. After his arrival in metropolitan France, a MAC organism further identified as genotype ST33 $M$. chimaera was isolated from his respiratory tract (Figure $1)$. This isolate was susceptible to clarithromycin $(3 \mu \mathrm{g} /$ $\mathrm{mL})$, rifabutin $(<1 \mu \mathrm{g} / \mathrm{mL})$, and ethambutol $(2 \mu \mathrm{g} / \mathrm{mL})$ and was resistant to ofloxacin $(>32 \mu \mathrm{g} / \mathrm{mL})$, streptomycin $(>1024 \mu \mathrm{g} / \mathrm{mL})$, doxycyclin $(>256 \mu \mathrm{g} / \mathrm{mL})$, and rifampicin $(>32 \mu \mathrm{g} / \mathrm{mL})$. The patient was given rifabutin and clarithromycin, but he interrupted this treatment after two weeks. Six months later his sputum yielded acid-fast bacilli that were identified as genotype MST33 $M$. chimaera after culture. The same antibiotic regimen prescribed for six months yielded negative mycobacterial cultures. Two years later a recurrent fever and cough correlated with MST33 M. chimaera-positive cultures. The patient once again interrupted his therapy of rifabutin combined with clarithromycin, this time after four months. Twenty-two months later the patient's condition severely worsened, as he had developed chronic respiratory insufficiency exacerbated by bilateral pneumothorax, which required non-invasive ventilatory support. From this period onward, insulin therapy became necessary to achieve glycemic control, and his nutritional condition justified the initiation of enteral feeding by percutaneous gastrostomy. The thoracic computed tomographic scan showed bronchiectasis, expanded areas of condensation, and mediastinal polyadenopathy. Acid-fast bacilli that were observed on sputum smears were cultured and revealed to be MST33 M. chimaera organisms. Rifabutin and clarithromycin were reintroduced, but sequential cultures remained positive. The patient was registered in the national list of lung transplant candidates.

During the whole treatment period, the microbiological investigations of the respiratory tract specimens also exhibited Pseudomonas aeruginosa, methicillin- susceptible Staphylococcus aureus, Stenotrophomonas maltophilia, and Aspergillus fumigatus.

\section{Discussion}

The prevalence of MAC organisms in patients with CF is highly variable, ranging from $9 \%$ in the US [1] to $1.5 \%$ in France, and it was evaluated at $1.8 \%$ in southeast France [5], where Marseilles is located, although our data yielded a prevalence of $4.3 \%$ there. In France and the US, $M$. avium has contributed to twice as many positive samples as $M$. intracellulare [5,7]. However, the identification of MAC organisms has relied on hybridization probe-based methods, which has misidentified $M$. chimaera as $M$. intracellulare [3], thus preventing the detection of $M$. chimaera in patients with CF. Using sequence-based identification, we observed that $M$. chimaera organisms contribute equally to respiratory tract infections in patients with CF compared with $M$. avium subsp. hominissuis isolates.

The pathogenicity of $M$. chimaera remains controversial. It has been reported to cause respiratory infections in patients with pulmonary diseases other than CF, including chronic obstructive pulmonary disease, bronchiectasis, and cavitation [3]. Conversely, in two studies with an aggregate of 97 non-CF patients OK, only $3.3 \%$ infected with $M$. chimaera fulfilled the American Thoracic Society (ATS) criteria for MAC lung disease $[8,9]$. The patient described in our case report eventually fulfilled the ATS criteria on the basis of positive cultures from at least two separate sputum samples. Moreover, his $M$. chimaera infection relapsed every time antibiotic treatment was interrupted, and this continuing infection correlated with a worsening condition of the patient in the presence of associated pathogens, which may have also contributed to this downward spiraling evolution [10].

\section{Conclusion}

We believe that $M$. chimaera should be regarded as an emerging opportunistic respiratory pathogen in patients with CF, including young children, and that its detection

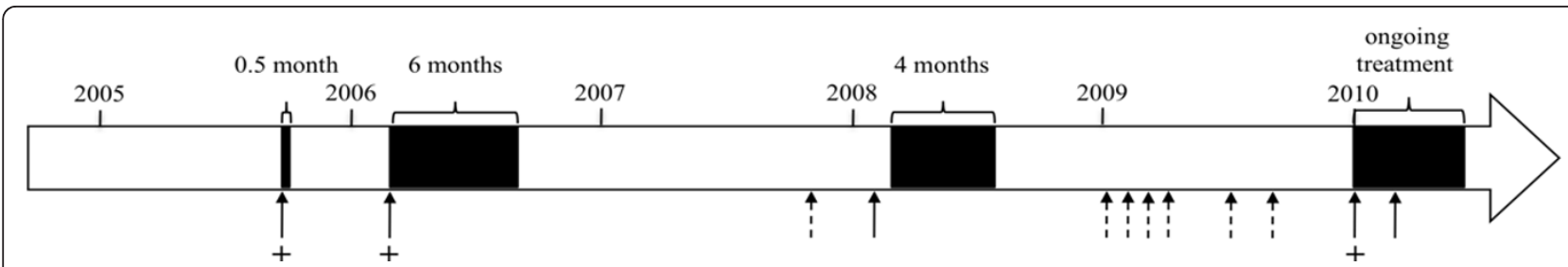

Figure 1 Chronological representation of Mycobacterium chimaera respiratory tract infection in a CF patient. Arrows indicate requests for mycobacterial detection in the sputum. Dotted arrows indicate negative culture. Filled arrows indicate positive culture for M. chimaera. + indicates acid-fast bacilli stain seen upon direct microscopic examination of sputum samples. Antibiotic therapy is represented by black squares. Duration of treatment (in weeks) is indicated above the brackets. 
warrants long-lasting, appropriate antimycobacterial treatment to eradicate it.

\section{Consent}

Written informed consent was obtained from the patient's mother for publication of this case report and any accompanying images. A copy of the written consent is available for review by the Editor-in-Chief of this journal.

\section{Abbreviations}

ATS: American Thoracic Society; CF: cystic fibrosis; MAC: Mycobacterium avium complex; MST: multi-spacer sequence typing.

\section{Acknowledgements}

This work was supported by Pôle des Maladies Infectieuses, Assistance Publique-Hôpitaux de Marseille, Marseille, France.

\section{Author details}

'Unité de Recherche sur les Maladies Infectieuses et Tropicales Emergentes, CNRS-UMR 6236, IRD 198, IFR 48, Faculté de Médecine, Université de la Méditerranée, 27, Boulevard Jean Moulin, Marseille cedex 5, France. ${ }^{2}$ Assistance Publique des Hôpitaux de Marseille-Pôle des Maladies Infectieuses, Hôpital la Timone, rue Saint-Pierre 13005 Marseille, France. ${ }^{3}$ Département des Maladies Respiratoires, Centre de Ressources et de Compétences pour la Mucoviscidose (CRCM), Hôpital Timone Enfants, rue Saint-Pierre 13005 Marseille, France.

\section{Authors' contributions}

$S C B, J M R, J C D$, and MD reviewed the clinical data and were major contributors to the writing of the manuscript. MD and NS were involved with patient management. SCB, JMR, and MD analyzed data and performed the laboratory analysis. All authors read and approved the final manuscript.

\section{Competing interests}

The authors declare that they have no competing interests.

Received: 22 March 2011 Accepted: 22 September 2011

Published: 22 September 2011

\section{References}

1. Olivier KN, Weber DJ, Lee JH, Handler A, Tudor G, Molina PL, Tomashefski J, Knowles MR, Nontuberculous Mycobacteria in Cystic Fibrosis Study Group: Nontuberculous mycobacteria. II: nested-cohort study of impact on cystic fibrosis lung disease. Am J Respir Crit Care Med 2003, 167:835-840.

2. Ben Salah I, Adekambi T, Raoult D, Drancourt M: rpoB sequence-based identification of Mycobacterium avium complex species. Microbiology 2008, 154:3715-3723.

3. Tortoli E, Rindi L, Garcia MJ, Chiaradonna P, Dei R, Garzelli C, Kroppenstedt RM, Lari N, Mattei R, Mariottini A, Mazzarelli G, Murcia MI, Nanetti A, Piccoli P, Scarparo C: Proposal to elevate the genetic variant MAC-A, included in the Mycobacterium avium complex, to species rank as Mycobacterium chimaera sp. nov. Int I Syst Evol Microbiol 2004, 54:1277-1285.

4. Turenne CY, Wallace R Jr, Behr MA: Mycobacterium avium in the postgenomic era. Clin Microbiol Rev 2007, 20:205-229.

5. Roux AL, Catherinot E, Ripoll F, Soismier N, Macheras E, Ravilly S, Bellis G, Vibet MA, Le Roux E, Lemonnier L, Gutierrez C, Vincent V, Fauroux B, Rottman M, Guillemot D, Gaillard JL, Jean-Louis Herrmann for the OMA Group: Multicenter study of prevalence of nontuberculous mycobacteria in patients with cystic fibrosis in France. J Clin Microbiol 2009, 47:4124-4128.

6. Cayrou C, Turenne C, Behr MA, Drancourt M: Genotyping of Mycobacterium avium complex organisms using multispacer sequence typing. Microbiology 2010, 156:687-694.

7. Olivier KN, Weber DJ, Wallace RJ Jr, Faiz AR, Lee JH, Zhang Y, BrownElliot BA, Handler A, Wilson RW, Schechter MS, Edwards LJ, Chakraborti S,
Knowles MR, Nontuberculous Mycobacteria in Cystic Fibrosis Study Group: Nontuberculous mycobacteria. I: multicenter prevalence study in cystic fibrosis. Am J Respir Crit Care Med 2003, 167:828-834.

8. Griffith DE, Aksamit T, Brown-Elliott BA, Catanzaro A, Daley C, Gordin F, Holland SM, Horsburgh R, Huitt G, lademarco MF, Iseman M, Olivier K, Ruoss S, von Reyn CF, Wallace RJ Jr, ATS Mycobacterial Diseases Subcommittee, American Thoracic Society, Infectious Disease Society of America: An official ATS/IDSA statement: diagnosis, treatment, and prevention of nontuberculous mycobacterial diseases. Am J Respir Crit Care Med 2007, 175:367-416.

9. Schweickert B, Goldenberg O, Richter E, Göbel UB, Petrich A, Buchholz P, Moter A: Occurrence and clinical relevance of Mycobacterium chimaera sp. nov., Germany. Emerg Infect Dis 2008, 14:1443-1446.

10. Bittar F, Richet H, Dubus JC, Reynaud-Gaubert M, Stremler N, Sarles J, Raoult D, Rolain JM: Molecular detection of multiple emerging pathogens in sputa from cystic fibrosis patients. PLoS One 2008, 3:e2908.

doi:10.1186/1752-1947-5-473

Cite this article as: Cohen-Bacrie et al: Mycobacterium chimaera pulmonary infection complicating cystic fibrosis: a case report. Journal of Medical Case Reports 2011 5:473.

\section{Submit your next manuscript to BioMed Central and take full advantage of:}

- Convenient online submission

- Thorough peer review

- No space constraints or color figure charges

- Immediate publication on acceptance

- Inclusion in PubMed, CAS, Scopus and Google Scholar

- Research which is freely available for redistribution

Submit your manuscript at www.biomedcentral.com/submit 